\title{
Therapie der Hypophosphatasie
}

\section{Treatments for Hypophosphatasia}

\section{(우(1) $(2)$}

Autoren

Lothar Seefried ${ }^{1}$, Franca Genest ${ }^{1}$, Uwe Kornak ${ }^{2}$, Oliver Semler ${ }^{3}$, Florian Barvencik ${ }^{4}$, Christine Hofmann ${ }^{5}$

Institute

1 Klinische Studieneinheit, Orthopädische Klinik - KLH, Universität Würzburg, Deutschland

2 Institut für Humangenetik, Universitätsmedizin Göttingen, Deutschland

3 Universität zu Köln, Medizinische Fakultät und Uniklinik Köln, Klinik und Poliklinik für Kinder- und Jugendmedizin, Köln, Deutschland

4 Institut für Osteologie und Biomechanik, Universitätsklinikum Hamburg-Eppendorf, Hamburg, Deutschland

5 Kinderklinik und Poliklinik, Universitätsklinikum Würzburg, Deutschland

Schlüsselwörter

Hypophosphatasie, Multimodale Behandlungsstrategie, Enzymersatz, Asfotase Alfa

Keywords

Hypophosphatasia, Multimodal Treatment Strategy, Enzyme Replacement Therapy, Asfotase Alfa

Bibliografie

Osteologie 2020; 29: 275-282

DOI 10.1055/a-1257-7976

ISSN 1019-1291

(c) 2020. The Author(s).

This is an open access article published by Thieme under the terms of the Creative Commons Attribution-NonDerivative-NonCommercial-License, permitting copying and reproduction so long as the original work is given appropriate credit. Contents may not be used for commercial purposes, or adapted, remixed, transformed or built upon.

(https://creativecommons.org/licenses/by-nc-nd/4.0/)

Korrespondenzadresse

Dr. med. Lothar Seefried

Klinische Studieneinheit Orthopädische Klinik - KLH

Universität Würzburg

Brettreichstraße 11, 97074 Würzburg, Deutschland

Tel.: +49 (0) 931/803-3575

Fax: +49 (0) 931/803-15 98

E-Mail: I-seefried.klh@uni-wuerzburg.de

\section{ZUSAMMENFASSUNG}

Die Hypophosphatasie (HPP) als Folge einer genetisch bedingt defizienten Aktivität der gewebeunspezifischen alkalischen Phosphatase (TNAP) ist geprägt durch ein ausgesprochen weites Spektrum möglicher Manifestationen, sowohl hinsichtlich der Art der Symptomatik als auch bzgl. des Schweregrades der assoziierten Einschränkungen. Eine adäquate Behandlung erfordert insofern immer auch eine multimodale Herangehensweise unter spezieller Berücksichtigung der individuellen Ausprägung der Erkrankung.

Für Patienten, bei denen die Erkrankung im Kindesalter aufgetreten ist, steht in Europa zur Behandlung der Knochenmanifestation mit Asfotase alfa (Strensiq) eine Enzymersatztherapie zur Verfügung. Sowohl in den Zulassungsstudien als auch in der klinischen Anwendung zeigen sich bei schwer betroffenen Kindern prinzipiell sehr erfreuliche radiologische und funktionelle Verbesserungen und ein verbessertes Gesamtüberleben. Auch bei Erwachsenen mit zulassungsentsprechender Krankheitsausprägung zeigen erste Ergebnisse eine deutliche Verbesserung der krankheitsassoziierten Einschränkungen und funktionelle Verbesserungen. Inzwischen gibt es auch ermutigende Daten zur Sicherheit und Wirksamkeit von Asfotase alfa über mehrere Behandlungsjahre.

Während die oftmals als stark belastend empfundenen entzündlichen Schmerzen häufig gut auf eine intermittierende, bedarfsangepasste Behandlung mit NSAR ansprechen, sind im Hinblick auf die muskuloskelettale Gesamtgesundheit nachhaltige, langfristig ausgerichtete supportive Maßnahmen mit spezifischen Trainingskonzepten und einer alters- und bedarfsgerechten orthopädietechnischen Versorgung zu empfehlen. Das Potenzial diätetischer Maßnahmen zur Beeinflussung des Phosphat- und Vitamin-B6-Stoffwechsels bedarf sicher noch weiterer Untersuchungen. Bezüglich spezifisch knochenwirksamer Strategien sind primär antiresorptive Substanzen nach aktueller Datenlage kritisch zu betrachten, wohingegen osteoanabole Konzepte grundsätzlich möglich erscheinen.

Sinnvollerweise sollte die Gesamtheit aller therapeutischen Maßnahmen an einem Zentrum mit entsprechender Erfahrung koordiniert und überblickt werden, wobei ein Großteil der konkreten Maßnahmen im aktiven Austausch auch heimatnah umgesetzt werden kann. 


\section{ABSTRACT}

Hypophosphatasia due to genetically determined deficient activity of the tissue non-specific alkaline phosphatase (TNAP) is characterized by a wide spectrum of potential clinical manifestations, both, regarding the type of symptoms, as well as the severity of associated deficits. Appropriate treatment strategies should be built on a multimodal approach specifically considering individual disease manifestation.

For patients with disease onset before adulthood, enzyme replacement therapy with Asfotase alfa (Strensiq) is approved in Europe to treat the bone manifestation of the disease. Both, available data from clinical trials as well as clinical routine experience confirm basically encouraging results of that treatment in severely affected children with substantial improvement regarding radiographic and functional outcome parameters as well as overall survival. Even in adult patients with severe disease manifestation pursuant to the approval, first results confirm substantial amelioration of disease-specific deficits and functional improvements. Meanwhile, there is also data supporting safety and efficacy of long-term treatment Asfotase alfa over several years.

While inflammatory pain, which is typically perceived as being burdensome, can commonly be addressed successfully with NSAIDs on-demand, overall musculoskeletal health requires sustained, multimodal, supportive treatment strategies including exercise interventions as well as age and health state adjusted technical orthopedic support. The use and potential clinical impact of Phosphate and Vitamin B6 on the course of the disease requires further investigation. Current data regarding the use of bone-targeted compounds is critical in terms of antiresorptives while osteoanabolic treatment strategies appear feasible.

Ideally, the entirety of therapeutic measures should be coordinated and overlooked at an experienced center while individual tasks can preferably be accomplished at local facilities near the patient's home.

\section{Hintergrund}

Die Hypophosphatasie (HPP) ist eine genetisch bedingte Erkrankung aufgrund einer Vielzahl unterschiedlicher genetischer Varianten im ALPL-Gen auf Chromosom 1p36.12 (OMIM\#171760), das für die gewebeunspezifische alkalische Phosphatase (TNAP) kodiert.

Während pathogene Veränderungen auf beiden Allelen im Sinne einer autosomal-rezessiven Erkrankung häufig mit sehr deutlichen Einschränkungen der Enzymaktivität und einer schwerwiegenden, frühzeitig klinisch manifesten Symptomatik einhergehen, sind heterozygote, monoallelische Veränderungen oftmals mit einer subtileren, klinisch weniger auffälligen Manifestation assoziiert [1-4]. Allerdings sind individuelle Verläufe auch über die Lebenszeit hinweg durchaus variabel und können insofern in beide Richtungen von dieser orientierenden Regel stark abweichen. Daher ist auch die traditionelle Einteilung des Schweregrades der Erkrankung anhand des Alters der Erstmanifestation [5] im klinischen Alltag nur wenig hilfreich, und es gilt, auf Grundlage eines empirisch inzwischen recht gut etablierten Repertoires an Behandlungsoptionen die optimale Therapie für die individuelle Patientensituation zu entwickeln.

Die im physiologischen Kontext nahezu ubiquitäre Expression des Enzyms in unterschiedlichen Geweben und seine zentrale Bedeutung bei der Spaltung von Phosphorsäuremonoestern in einer Vielzahl von Stoffwechselvorgängen bietet zahlreiche, teils noch hypothetische Erklärungsmodelle für das breite Spektrum möglicher klinischer Manifestationen der Erkrankung. Recht gut erforscht sind die Pathophysiologie der skelettalen Mineralisationsstörung durch die Akkumulation von anorganischem Pyrophosphat (PPi) und Phospho-Osteopontin sowie die Rolle der ALP für die Dephosphorylierung von Pyridoxalphosphat (PLP), der zirkulierenden Hauptform von Vitamin B6. Weiterer Untersuchungen bedarf demgegenüber die Klärung des Einflusses der ALPDefizienz auf purinerge Signalwege, die Interkonversion von Neurotransmittern, die Relevanz für den Energieumsatz und die Metabolisierung energiereicher Triphosphate. In diesem Kontext ebenfalls noch offen sind Fragen der Bedeutung der Hypophosphatasie für den Energiestoffwechsel und die Geweberegeneration, wie auch die klinische Signifikanz der gewebeunspezifischen ALP für die Detoxifikation bakterieller Lipopolysaccharide. Pathophysiologisch wesentlich und noch unzureichend verstanden sind darüber hinaus auch die Mechanismen, über die ein Mangel an ALP-Aktivität und die konsekutive Akkumulation von Metaboliten über direkte, rezeptorvermittelte und/oder indirekte, gewebeassoziierte Effekte eine klinisch entzündlich imponierende Symptomatik induzieren können.

Jenseits dieser pathophysiologisch-wissenschaftlichen Herausforderungen, deren Klärung perspektivisch sicher auch entscheidende Erkenntnisse für eine Optimierung der Behandlungsmöglichkeiten liefern wird, erfordert das pleiotrope Spektrum klinischer Krankheitsmanifestationen bereits heute therapeutische Antworten. Eine Übersicht über häufige Krankheitszeichen und Symptome, die von den Patienten berichtet werden und die damit auch als zentrale Herausforderung für die Behandelnden zu sehen sind, gibt $>$ Abb. 1. Für Details dazu wie auch zum Vorgehen und zu den Herausforderungen im Rahmen der Diagnostik sei auf entsprechende Vorarbeiten verwiesen [69]. Diese Übersicht fokussiert auf therapeutische Möglichkeiten, die wir nach heutigem Kenntnisstand zur Verfügung haben und den Patienten anbieten können.

\section{Enzymersatztherapie}

Seit der Zulassung einer Langzeit-Enzymersatztherapie (ERT) Asfotase alfa (Strensiq) im Herbst 2015 zur Behandlung der Knochenmanifestation bei Patienten, bei denen die HPP im Kindesund Jugendalter aufgetreten ist, steht erstmals ein kausaler Therapieansatz gerade für diese in der Regel komplexeren Verläufe der HPP zur Verfügung [10]. Bei Asfotase alfa handelt es sich um das rekombinante humane Enzym, gekoppelt an ein $\mathrm{IgG}_{1}-\mathrm{Fc}$ Fragment sowie einen Deca-Aspartat-Knochenanker, der durch 


Zähne:
- Vorzeitiger Zahnverlust
- $\quad$ von Milchzähnen/bleibenden Zähnen
- $\quad$ Karies

Muskulär:

- Muskelschwäche-/Schmerzen

- Ermüdbarkeit

- Induration / Myogelosen

$$
\begin{aligned}
& \text { Gelenke: } \\
& \text { - Arthritis } \\
& \text { - Chondrokalzinose } \\
& \text { - Tendinosis calcarea/Sehenverkalkung } \\
& \text { - Pseudogicht }
\end{aligned}
$$

\section{Knochen:}

- Pseudo-/Insuffizienzfrakturen Loosersche Umbauzonen

- Knochenmarködeme

- Osteomalazie
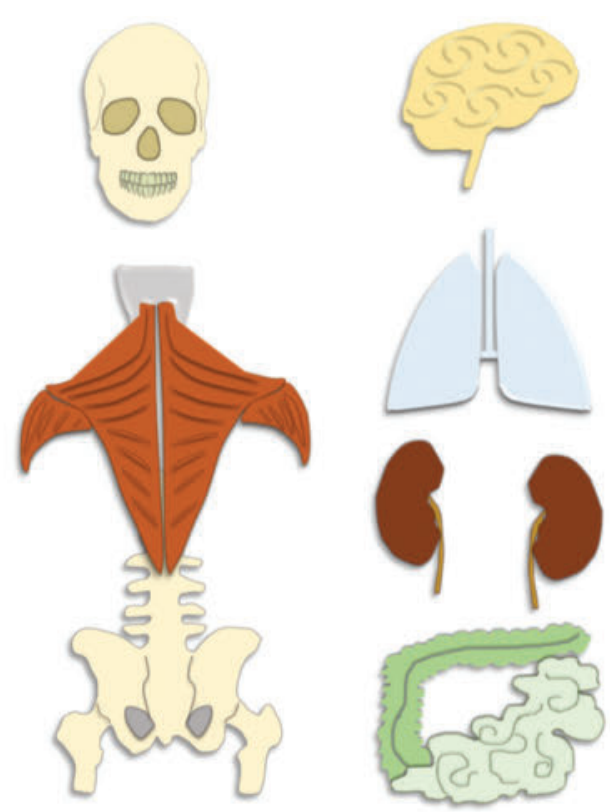

ZNS / Neurologisch:

- Vit B6 - abhängige Krämpfe

- Kraniosynostose

- Cephalgie / Migrăne

- Ängstlichkeit,Nervosität

- Depressive Verstimmung

Respiratorsich:

- Respiratorische Insuffizienz

- Lungenhypoplasie

- Beatmungspflichtigkeit

\section{Niere: \\ - Nephrokalzinose \\ - Nierenfunktionsstörungen \\ - Nierensteine}

\section{Intestinal:}

- Diarrhoe / Obstipation

- Meteorismus

- Unverträglichkeiten

- Abb 1. Spektrum häufiger Organmanifestationen bei Hypophosphatasie

- Fig. 1 Spectrum of common clinical manifestation of Hypophosphatasia

Bindung an das Calciumhydroxylapatit des Knochens den Verbleib und die Wirksamkeit des Enzyms am Knochen sichert. Die Applikation erfolgt subkutan gewichtsadaptiert in einer empfohlenen Dosierung von $6 \mathrm{mg} / \mathrm{kg}$ Körpergewicht (KG) pro Woche, verteilt auf 3 bzw. 6 wöchentliche Gaben à 2 mg/kg bzw. 1 mg/ kg KG. Aktuelle Untersuchungen legen nahe, dass diese primär bei Kindern etablierte Größenordnung der Dosierung auch bei erwachsenen Patienten adäquat zu sein scheint.

Die der Zulassung zugrunde liegenden Studienergebnisse belegen für die Enzymersatztherapie insbesondere bei lebensbedrohlich betroffenen Kindern mit perinataler bzw. infantiler HPP (Krankheitsbeginn bis zum 6. Lebensmonat) ein erfreuliches muskuläres und radiologisches Ansprechen, eine respiratorische Verbesserung sowie insbesondere ein verbessertes Gesamtüberleben im Vergleich zum natürlichen Verlauf der Erkrankung in einer historischen Kohorte [11-14]. Die Analyse dieser Daten im Rahmen der nationalen Vorgaben zur Nutzenbewertung von Arzneimitteln durch den gemeinsamen Bundesausschuss (G-BA) bestätigt diesbezüglich auch einen Zusatznutzen der Therapie im Hinblick auf die Mortalität bei Kleinkindern ( $\leq 5$ Jahre) mit Krankheitsbeginn bis zum 6. Lebensmonat. Dies impliziert auch, dass ein Zusatznutzen der Therapie für andere Zielparameter und Patientengruppen nach den geltenden formalen Kriterien nicht als pauschal belegt angenommen werden kann. Gleichwohl zeigen die wissenschaftlichen Daten, dass im Einzelfall bei schwer betroffenen Patienten durchaus ein konkret fassbarer (Zusatz-)Nutzen durch die Therapie erzielt werden kann. Wesentlich scheint hier eine differenzierte, patientenindividuelle Abwägung von Chancen und Risiken, um die Patienten zu identifizieren, die von einer ERT nachweislich profitieren. So konnten in einer Studie in der Tat auch bei älteren Kindern (6-12 Jahre, $\mathrm{n}=12$ ) ein radiologisches Ansprechen sowie eine Verbesserung von Muskelkraft und Wachstum sowie der motorischen Fähigkeiten und damit einhergehend eine verbesserte Lebensqualität im Vergleich zu historischen Kontrollen nachgewiesen werden [15]. Auch Daten zur Enzymersatztherapie bei Jugendlichen und Erwachsenen mit histologisch nachgewiesener Osteomalazie (13-65 Jahre, $n=19$ ) zeigen bei adäquater Dosierung das erreichbare Verbesserungspotenzial, wenngleich die individuelle Variabilität des Ansprechens in dieser Studie auch die Notwendigkeit einer sorgfältigen Auswahl der Patienten unterstreicht [16]. Auch diverse Kasuistiken untermauern die erfreuliche Regeneration des Knochens unter der Therapie auch bei erwachsenen Patienten [17-19], und selbst bei lange bestehenden Looserschen Umbauzonen zeigt sich unter der Therapie eine erfreuliche Durchbauung ( $\triangleright$ Abb. 2). Erste Auswertungen der 12-Monats-Daten von 14 Teilnehmern (19-78 Jahre) einer längerfristig angelegten Therapieverlaufsstudie bei schwer betroffenen erwachsenen Patienten (EmPATHY) mit kindlicher Erstmanifestation zeigen ebenfalls signifikante funktionelle Verbesserungen, insbesondere hinsichtlich der Parameter zur Überprüfung der Alltagsmobilität, wie etwa dem Chair-Rise Test und dem 6-Minute Walk Test [20]. Interessant wird sein, im Verlauf zu verstehen, inwiefern dies wesentlich mit einer verbesserten Belastbarkeit des Knochens assoziiert ist oder aber auch indirekte Effekte auf die Muskulatur und andere Stoffwechselprozesse eine ursächliche Rolle spielen, etwa durch Veränderungen der Konzentration von Substraten oder durch zirkulierendes Enzym. Dies impliziert auch die Frage nach möglichen unerwünschten Effekten der Therapie, nicht zuletzt in extraskelettalen Geweben. Bedingt durch die 3-maligen bzw. 6-maligen wöchentlichen Injektionen sind trotz der empfohlenen Rotation zwischen verschiedenen 

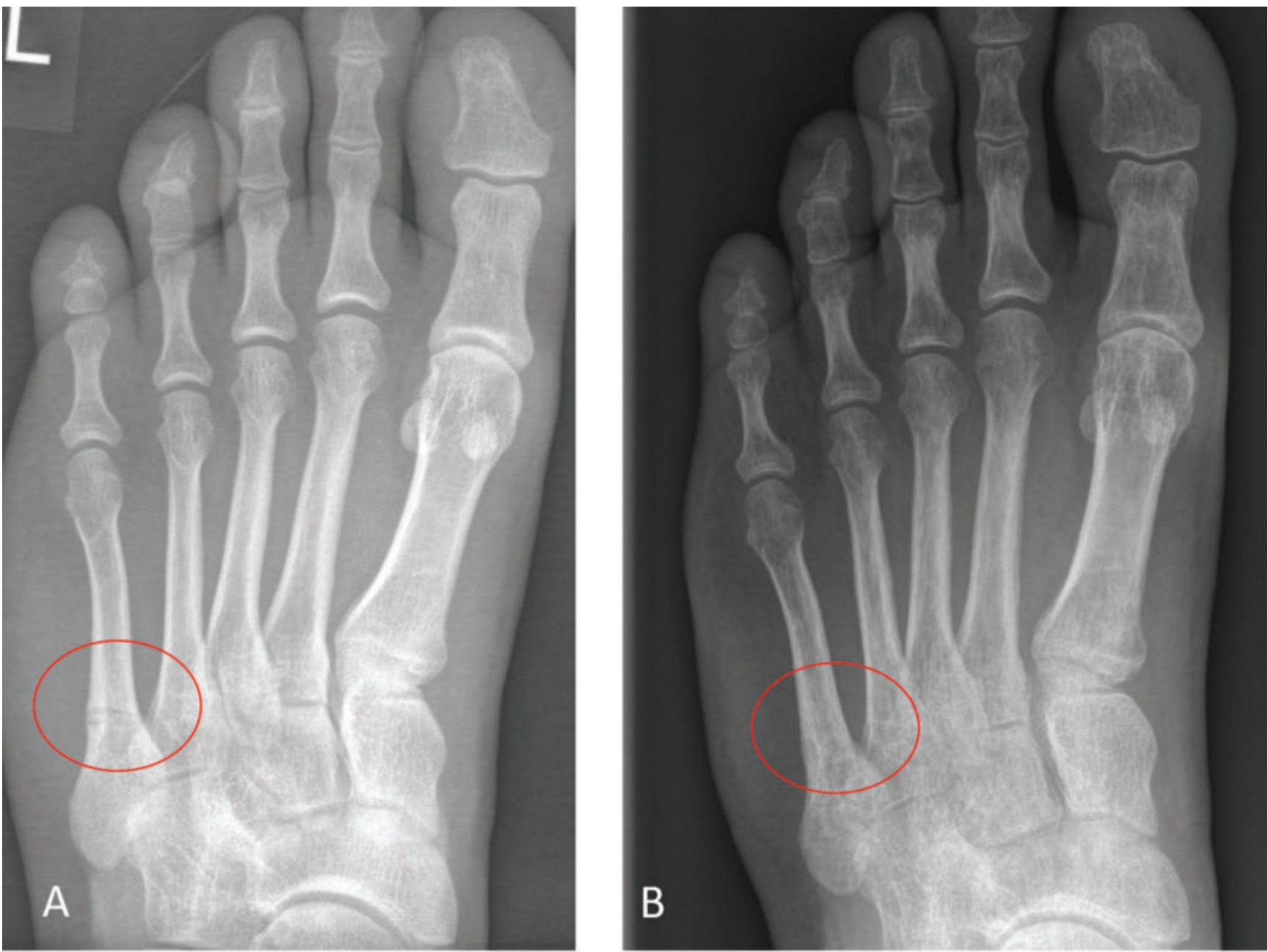

- Abb 2. A) Bild einer nicht heilenden MT-5-Fraktur bei compound heterozygot betroffenem Patienten mit frühkindlicher Erstmanifestation (Kraniosynostose); B) Konsolidierung 3 Monate nach Beginn der Enzymersatztherapie

-Fig. 2 A) X-ray of a non-healing fracture of the fifth metatarsal bone in a compound heterozygously affected patient with infantile onset (Craniosynostosis) B Osseous consolidation 3 months after initiation of enzyme replacement therapy.

Injektionsstellen Veränderungen im Bereich der Einstichstellen mit einer teilweisen Verfärbung und der Entstehung einer Lipodystrophie nicht selten. Beobachtet werden im Einzelfall auch anaphylaktoide Reaktionen sowie Laborwertveränderungen mit einem transienten Anstieg des PTH in den ersten Wochen und Monaten und vereinzelt Verminderungen des Kalziumspiegels. Regelmäßige Kontrollen auf ektope Kalzifikationen alle 6 Monate, insbesondere im Bereich der Nieren und der Augen [21] sowie bei Kindern im Hinblick auf die Entstehung einer Kraniosynostose, gehören zum allgemeinen Standard in der Verlaufsbeobachtung, während Kontrollen auf Kalzifikationen der großen Gefäße aufwändiger sind und daher individuell indiziert werden sollten, insbesondere bei kardiovaskulären Risikopatienten. Die Frage der klinischen Signifikanz der Bildung von Antikörpern gegen das rekombinante Enzym, sog. Anti-Drug Antibodies (ADA), sowie vereinzelt beschriebene, mögliche Veränderungen der Wirksamkeit im langfristigen Therapieverlauf sind Gegenstand aktueller Forschungen. Dahingehend wie auch zur Frage des langfristigen Nutzens können uns perspektivisch die laufenden Studien sowie das globale HPP-
Register (NCT02306720) wertvolle Informationen liefern, und eine entsprechende Anbindung der Patienten ist sicher sinnvoll. Es ist wesentlich, gerade bei Patienten unter Enzymersatztherapie eine interdisziplinäre Betreuung zu gewährleisten und bereits zu Therapiebeginn klare Behandlungsziele zu definieren, deren Erreichen auch regelmäßig kontrolliert werden sollte - als Grundlage für eine verantwortungsvolle individuelle Nutzen-Risiko-Abwägung $[22,23]$.

Jenseits der sehr positiven Daten zur Wirksamkeit der Enzymersatztherapie im Hinblick auf die unterliegende Knochenpathologie und potenzielle günstige Off-target-Effekte verbleiben auch bei Patienten unter ERT weitere behandlungsbedürftige Probleme. Vor dem Hintergrund der Datenlage und der darauf basierenden Zulassungssituation qualifizieren auch bei weitem nicht alle Patienten mit einer Variante im ALPL-Gen für eine Enzymersatzbehandlung bzw. werden davon nicht dauerhaft profitieren. Insofern kommt der interdisziplinären, multimodalen, symptomatischen Behandlung dieser Herausforderungen große Bedeutung zu. Dies betrifft insbesondere eine adäquate Betreuung bzgl. der 


\section{Bausteine der HPP- Therapie}

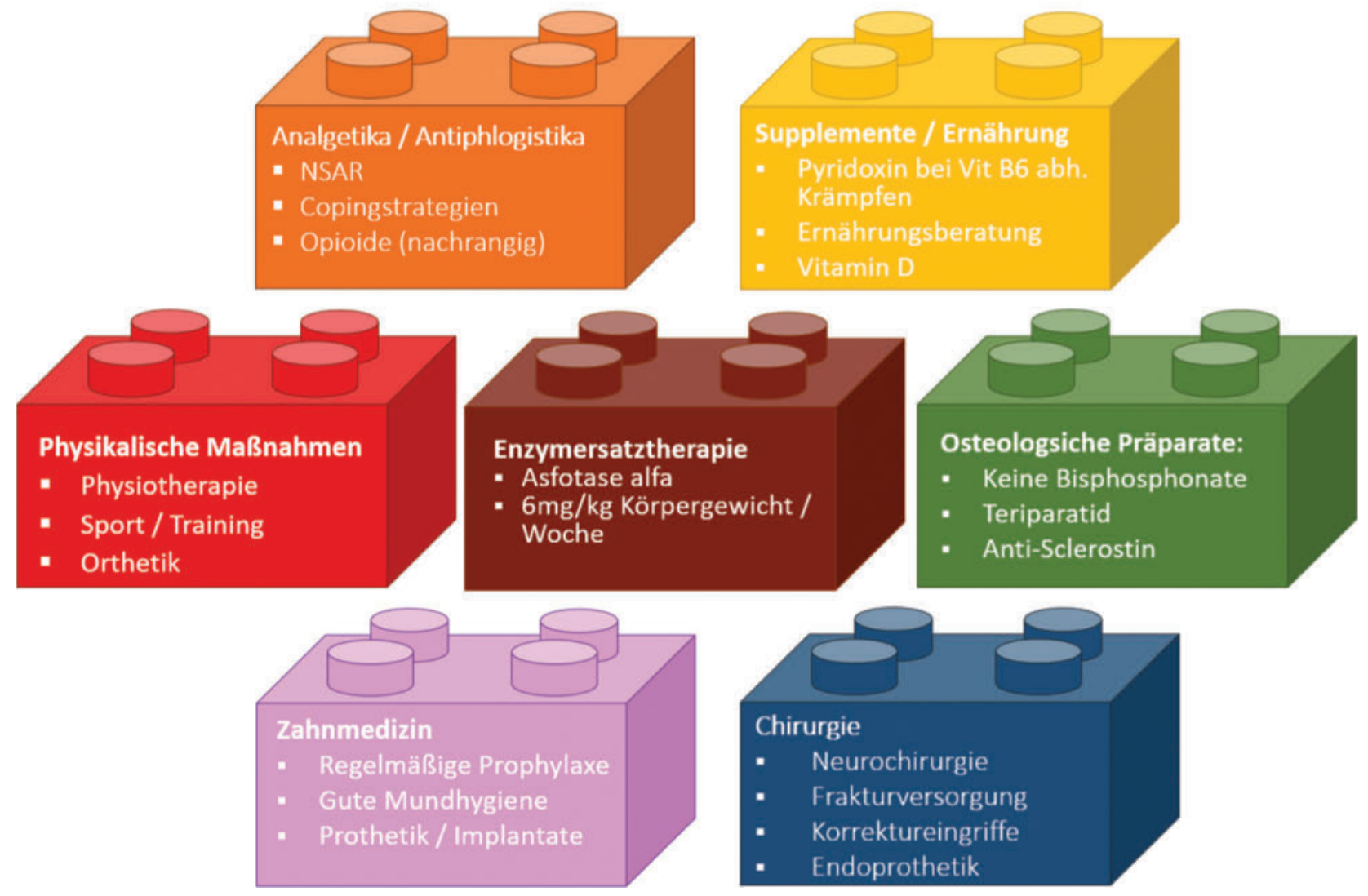

Abb 3. Bausteine der HPP-Therapie

-Fig. 3 Essential components of HPP Treatment

Schmerzen, der funktionellen muskuloskelettalen Probleme, der reduzierten Leistungsfähigkeit und Erschöpfung, die Behandlung der Zahnprobleme und das Monitoring der Nierenfunktion sowie die Therapie von neurologischen, psychologischen und neuropsychiatrischen Problemen.

\section{Bewegung, Sport und Physiotherapie}

Körperliche, vorwiegend muskulär empfundene Erschöpfung und eine begleitende Schwäche sind ein häufig von den Patienten beklagtes Phänomen [24]. Im Kontext des kompromittierten Phosphatstoffwechsels scheint es denkbar, dass auch die Energiebereitstellung im Muskel betroffen ist, was sowohl eine eingeschränkte (Ausdauer-)Belastbarkeit als auch eine protrahierte Rekonvaleszenz der Patienten nach Belastung erklären könnte [25, 26]. Gleichzeitig sind die Entwicklung von Mobilität und Kraft im Kindesalter bzw. der Erhalt der körperlichen Leistungsfähigkeit im Erwachsenenalter zentrale Determinanten für die von den Patienten empfundene Lebensqualität bzw. die Belastung durch die Erkrankung [27, 28]. Physiotherapie und medizinische
Trainingstherapie unter fachkundiger Anleitung und mit einer instruktiven Komponente zum Training in Eigenregie scheinen vor diesem Hintergrund empirisch gute Möglichkeiten, diesem Ziel ohne wesentliche Risiken und unerwünschte Begleiterscheinungen näher zu kommen [29]. Angesichts der bei Hypophosphatasie überproportional häufig beklagten muskulären Indurationen und Verspannungen, vor allem in der Nacken-Schulter-Region, sollte die Therapie von detonisierenden Maßnahmen (manuelle Therapie, Wärmetherapie etc.) begleitet werden, um einen muskulären Hypertonus und damit assoziierte Schmerzen im Sinne eines Circulus vitiosus möglichst zu vermeiden [30, 31].

Sportliche Betätigungen, gerade auch zum Aufbau einer stabilen Muskulatur im Kindes- und Jugendalter, sind grundsätzlich sinnvoll, wobei hier die spielerische Komponente und der Spaß nicht zu kurz kommen sollten, um eine dauerhafte Eigenmotivation zu bahnen. Gerade ausdauernd monotone Belastungsformen oder eine individuelle Überbelastung scheinen bei vielen HPPPatienten mit Schmerzen und einer prolongierten Erschöpfung einherzugehen und sind insofern auch unter Motivationsaspekten oftmals kontraproduktiv. 
Die Erfahrungen mit der Enzymersatztherapie zeigen, dass muskuläre Probleme, die wesentlich zu den motorischen Defiziten bei Kindern und der Trainingsintoleranz bei Erwachsenen beitragen, unter der Enzymersatztherapie individuell gebessert werden können, wobei dieser Effekt interindividuell inkonsistent, im Therapieverlauf variabel und pathophysiologisch nicht abschließend erklärbar erscheint. Grundsätzlich sollten Trainingsmaßnahmen daher entsprechend der persönlichen Belastbarkeit geplant werden und sind dann auch unabhängig von der Frage einer ERT oder dem Lebensalter für alle Patienten sinnvoll und empfehlenswert.

\section{Orthopädietechnische Versorgung}

Gerade bei den schwer betroffenen Patientinnen und Patienten mit einer wesentlichen skelettalen Manifestation und rachitischen Veränderungen im Kindesalter erfordern die Deformierungen und Achsabweichungen im Bereich der Wirbelsäule, der Beine und der Füße sowie die Residuen stattgehabter Frakturen bei gleichzeitiger muskulärer Schwäche eine kompetente orthopädietechnische Begleitung. Eine kluge Ausschöpfung des noch gegebenen Korrekturpotenzials im Wachstumsalter kann helfen, spätere operative Eingriffe und Spätschäden einer Deformierung zu vermeiden. Gleichzeitig können supportive orthopädietechnische Maßnahmen helfen, in jedem Alter die funktionellen Einschränkungen vorliegender Behinderungen auf den Alltag der Betroffenen gering zu halten.

\section{Supplemente und Ernährung}

Bereits in der perinatalen Situation ergibt sich bei den schweren Verlaufsformen mit zerebralen Krampfanfälle die Indikation für eine Behandlung mit hochdosiertem Pyridoxin (nicht phosphoryliertes Vitamin B6). Zu Grunde liegt die Annahme, dass es im Zuge der gestörten Dephosphorylierung von Pyridoxalphosphat (PLP), das in dieser Form die Plasmamembran bzw. die Blut-HirnSchranke nicht überschreiten kann, zu einem intrazellulären Mangel an Vitamin B6 und einer gestörten Konversion von Neutrotransmittern kommt und dadurch die Anfallsschwelle im Gehirn herabgesetzt wird.

Darüber hinaus findet sich bei schwer betroffenen Säuglingen und Kleinkindern häufig eine Gedeihstörung, teilweise verbunden mit Übelkeit und nutritiven Schwierigkeiten. Dahingehend kommt einer adäquaten, altersgerechten Ernährung, ggf. auch mit Unterstützung einer professionellen Ernährungsberatung, eine wesentliche Rolle zu. Nicht selten ist bei diesen Patienten, zumindest intermittierend, auch eine enterale Ernährung über eine Sonde erforderlich, um ein entsprechendes Gedeihen der Kinder zu erreichen.

Eine Ernährung mit kalzium- bzw. phosphatarmer Kost bzw. der Einsatz von Phosphatbindern erscheinen bei Hyperkalzämie bzw. -phosphatämie sinnvoll. Bei ausgeprägter Hyperkalzämie im Säuglingsalter können zusätzliche medikamentöse Maßnahmen erforderlich sein (z. B. intravenöse Flüssigkeitsgabe, Furosemid, ggf. kurzfristig Glukokortikosteroide etc.). Bei erwachsenen Patienten konnte in einer Querschnittserhebung gezeigt werden, dass nicht nur eine sehr hohe, sondern auch eine sehr niedrige alimentäre Phosphatzufuhr, aber auch eine defiziente bzw. eine gesteigerte Kalziumzufuhr mit vermehrt klinischen Symptomen einhergehen gegenüber einer durchschnittlichen Phosphataufnahme, idealerweise in Kombination mit einem balancierten Kalzium-Phosphor-Verhältnis. Darüber hinaus gab es in dieser wie auch in einer früheren Untersuchung bisher keinen Beleg für einen therapeutischen Nutzen einer gesteigerten alimentären Zufuhr von Zink und Magnesium als Kofaktoren des Enzyms [32, 33].

Eine Substitution mit Vitamin D orientiert sich bei Kindern wie auch bei den Erwachsenen an den Empfehlungen für Gesunde bzw. erfolgt abhängig vom entsprechenden Serumspiegel.

\section{Analgetisch-antiphlogistische Therapie}

Eine entzündliche muskuloskelettale Schmerzsymptomatik zählt zu den am weitesten verbreiteten Symptomen bei HPP-Patienten und betrifft insbesondere die großen Muskelgruppen der Oberschenkel und Wirbelsäule, wie auch die des Schultergürtels und Nackens [34]. In früheren Arbeiten konnte bei Kindern und Jugendlichen eine gute Wirksamkeit von NSAR bzgl. dieser Symptomatik gezeigt werden. Vor dem Hintergrund der vermuteten entzündlichen Schmerzgenese wie auch der von den Patienten nahezu schon rheumaartig anmutenden geschilderten Beschwerden scheint auch eine analgetische Therapie mittels NSAR sinnvoll und zielführend und damit Mittel der ersten Wahl. Grundsätzlich sollte der Einsatz dieser Präparate unter Berücksichtigung möglicher Nebenwirkungen jedoch nicht im Sinne einer kontinuierlichen Langzeittherapie, sondern lediglich bedarfsweise zur symptomatischen Linderung von Schmerzspitzen eingesetzt werden [35]. Im Falle einer Unverträglichkeit können gängige weitere Schmerzmittel genutzt werden, wobei Opioide erfahrungsgemäß nur eine vergleichsweise unzureichende Wirkung auf die HPP-typischen Schmerzen haben, was sich auch bei fortschreitender Dosiseskalation nicht grundsätzlich ändert.

\section{Spezifisch-osteologische Präparate}

Jenseits der HPP-bedingten Mineralisierungsstörungen finden sich gerade bei erwachsenen Patientinnen und Patienten mit pathogenetisch relevanten ALPL-Varianten Osteoporose-typische Frakturen, wie Sinterungsfrakturen der Wirbelkörper und metaphysäre Frakturen der Röhrenknochen, etwa am distalen Radius, am proximalen Femur (Schenkelhals, pertrochantäre Region) oder am proximalen Humerus in Verbindung mit einer erniedrigten Knochendichte. Angesicht der bei HPP krankheitsimmanent reduzierten Umbauaktivität mit pathognomonischer Akkumulation von Pyrophosphatverbindungen ist dabei eine Behandlung mit antiresorptiven Präparaten ganz allgemein und mit Bisphosphonaten im Besonderen sehr zurückhaltend zu indizieren bzw. als relativ kontraindiziert zu werten, zumal dadurch bei schwerwiegender Enzymdefizienz möglicherweise das Risiko für krankheitstypische Pseudofrakturen bzw. Loosersche Umbauzonen noch gesteigert werden könnte [36-38].

Der Einsatz von osteoanabolen Therapeutika erscheint demgegenüber grundsätzlich plausibel, und in der Tat gibt es 
inzwischen eine Reihe an publizierten und wohl noch mehr unpublizierte Kasuistiken, in denen der Einsatz von Teriparatid situativ zur Steigerung der Knochenumbauaktivität hilfreich oder zumindest nicht schädlich war. Leider sind die berichteten Effekte aber vergleichsweise inkonsistent und erwartungsgemäß nur transient, d.h., eine nachhaltige Wirksamkeit über die Dauer der eigentlichen Therapie hinaus ist nicht zu erwarten. Vor dem Hintergrund der limitierten Gesamttherapiedauer - gemessen auf die Lebenszeit - sollten Zeitpunkt bzw. Zeitraum einer solchen Behandlung differenziert abgewogen werden [39-42]. Für den Einsatz eines Sclerostin-Antikörpers konnte in einer Phase-II-Studie eine grundsätzliche Wirksamkeit dieses Prinzips bei HPP-Patienten im Sinne einer Verbesserung der Knochendichte und Steigerung der Knochenformationsaktivität bei gleichzeitig guter Verträglichkeit nachgewiesen werden, sodass dies eine weitere Option zur Behandlung bei koinzidenter Osteoporose und HPP bzw. bei Notwenigkeit zur Steigerung der Formation bei unterliegender HPP darstellt [43].

\section{Zahnmedizin}

Da zahnmedizinische Probleme eine der charakteristischen Manifestationen der HPP darstellen, ist auch eine lebenslange Anbindung an einer entsprechend versierten zahnmedizinischen Einrichtung empfehlenswert. Schon beim pathognomonischen frühzeitigen Verlust der Milchzähne sollte der Einsatz einer Kinderprothese erwogen werden, da dies wesentliche Auswirkungen auf die sprachliche Entwicklung und die schulisch-soziale Integration der Patientinnen und Patienten hat. Auch im weiteren Krankheitsverlauf bei häufig bekannt kompromittiertem Status der Zähne und des Zahnhalteapparates, trotz sorgfältiger Mundhygiene, stellt die kieferorthopädische und parodontologische Betreuung der Patienten eine wesentliche Therapiesäule dar. Korrekturen von Zahnfehlstellungen sollten mit einem in der Behandlung von HPP-Patienten erfahrenen Kieferorthopäden abgestimmt werden [44, 45].

\section{Chirurgische Maßnahmen}

Bei den schwer betroffenen Kindern mit perinataler bzw. infantiler Erkrankungsmanifestation ist der vorzeitige Verschluss der Schädelnähte im Sinne einer Kraniosynostose mit dem Risiko einer Verlagerung von Anteilen des Kleinhirns in den Rückenmarkskanal bzw. der Bildung von Flüssigkeitsspalten im Rückenmark im Rahmen einer intrakraniellen Druckerhöhung und Liquorzirkulationsstörung eine der wesentlichen Komplikationen. Patienten mit Kraniosynostosen bedürfen daher einer regelmäßigen neurologischen bzw. neurochirurgischen sowie ophthalmoskopischen und radiologischen Kontrolle, um den Zeitpunkt für eine erforderliche neurochirurgische Intervention zur Dekompression nicht zu versäumen. Klinisch wegweisend sind dabei neurologische Symptome, beispielsweise Kopfschmerzen, Lähmungen oder Taubheitsgefühl in den Armen, aber auch das Neuauftreten eines Papillenödems [46, 47].

Die operative Stabilisierung von Frakturen stellt insofern eine spezielle Herausforderung dar, als grundsätzlich gerade bei schwer betroffenen Pateinten von einer kompromittierten
Knochenqualität und einer prolongierten Heilungsdauer ausgegangen werden muss. Bei HPP-typischen Frakturen, die häufig als Folge latenter Pseudofrakturen im diaphysären Bereich der langen Röhrenknochen auftreten, scheint die Anwendung extramedullärer Kraftträger, wie etwa Platten, mit einem höheren Risiko für komplikative Verläufe assoziiert. Insofern sollte adäquat dimensionierten intramedullären Kraftträgern der Vorzug gegeben werden [38]. Auch für die endoprothetische Versorgung gelten ähnliche Prinzipien.

Sinnvollerweise sollte die Gesamtheit dieser Maßnahmen ( $\triangleright$ Abb. 3) an einem Zentrum mit entsprechender Erfahrung koordiniert und überblickt werden, was einer heimatnahen Umsetzung eines Großteils der Maßnahmen keineswegs entgegensteht. Im Gegenteil ist eine konstruktive Zusammenarbeit aller Beteiligten entscheidend für eine erfolgreiche multimodale und multidisziplinäre Behandlung im Sinne der Patienten.

\section{Funding Information}

Die Open Access Publikation dieses Artikels wurde von den Firmen Alexion, Kyowa Kirin und Takeda durch die Übernahme der APC ermöglicht. Es erfolgte keinerlei Einflussnahme dieser Firmen auf die Inhalte des Artikels.

Interessenkonflikt

L. Seefried erhielt Mittel für Forschungsprojekte von Alexion, Kyowa Kirin und Novartis sowie Honorare für Vorträge oder Beratung von Alexion, Amgen, Lilly, Kyowa Kirin und UCB.

\section{References}

[1] Mornet E, Hofmann C, Bloch-Zupan A et al. Clinical utility gene card for: Hypophosphatasia - update 2013. Eur J Hum Genet 2014; 22: 4. doi: 10.1038/ejhg.2013.177

[2] Millán JL, Whyte MP. Alkaline Phosphatase and Hypophosphatasia. Calcif Tissue Int. 2016; 98: 398-416. doi: 10.1007/s00223-015-0079-1

[3] Whyte MP. Hypophosphatasia - aetiology, nosology, pathogenesis, diagnosis and treatment. Nat Rev Endocrinol 2016; 12(4): 233-246

[4] Seefried L, Dahir K, Petryk A et al. Burden of Illness in Adults with Hypophosphatasia: Data from the Global Hypophosphatasia Patient Registry. J Bone Miner Res 2020. doi: 10.1002/jbmr.4130

[5] Fraser D. Hypophosphatasia. Am J Med 1957; 22(5): 730-746

[6] Högler W, Langman C, Gomes da Silva $\mathrm{H}$ et al. Diagnostic delay is common among patients with hypophosphatasia: Initial findings from a longitudinal, prospective, global registry. BMC Musculoskelet Disord 2019; 20(1): 80. doi: 10.1186/s12891-019-2420-8

[7] Whyte MP. Hypophosphatasia: An overview For 2017. Bone 2017; 102: $15-25$

[8] Hofmann C. Klinik und Diagnostik der Hypophosphatasie im Kindesalter. Osteologie 2017; 26(1): 32-35

[9] Seefried L, Genest F. Klinik und Diagnostik der Hypophosphatasie im Erwachsenenalter. Osteologie 2017; 26(1): 36-41

[10] Hofmann C, Seefried L, Jakob F. Asfotase alfa: Enzyme replacement for the treatment of bone disease in hypophosphatasia. Drugs Today (Barc) 2016; 52(5): 271-285 
[11] Whyte MP, Kishnani PS, Greenberg CR et al. Hypophosphatasia: Enzyme replacement therapy (asfotase alfa) decreases TNSALP substrate accumulation and improves functional outcomes in affected adolescents and adults. Bull Group Int Rech Sci Stomatol Odontol 2012; 51(1): e35

[12] Whyte MP, Rockman-Greenberg C, Ozono K et al. Asfotase Alfa Treatment Improves Survival for Perinatal and Infantile Hypophosphatasia. J Clin Endocrinol Metab 2016; 101(1): 334-342

[13] Whyte MP, Simmons JH, Moseley S et al. Asfotase alfa for infants and young children with hypophosphatasia: 7 year outcomes of a singlearm, open-label, phase 2 extension trial. Lancet Diabetes Endocrinol 2019; 7(2): 93-105

[14] Hofmann CE, Harmatz P, Vockley J et al. Efficacy and Safety of Asfotase Alfa in Infants and Young Children With Hypophosphatasia: A Phase 2 Open-Label Study. J Clin Endocrinol Metab 2019; 104(7): 2735-2747

[15] Whyte MP, Madson KL, Phillips D et al. Asfotase alfa therapy for children with hypophosphatasia. JCl Insight 2016; 1(9): e85971

[16] Kishnani PS, Rockman-Greenberg C, Rauch F et al. Five-year efficacy and safety of asfotase alfa therapy for adults and adolescents with hypophosphatasia. Bone 2019; 121: 149-162

[17] Rolvien T, Schmidt T, Schmidt FN et al. Recovery of bone mineralization and quality during asfotase alfa treatment in an adult patient with infantile-onset hypophosphatasia. Bone 2019; 127: 67-74

[18] Freitas TQ, Franco AS, Pereira RMR. Improvement of bone microarchitecture parameters after 12 months of treatment with asfotase alfa in adult patient with hypophosphatasia: Case report. Med (Baltimore) 2018; 97(48): e13210

[19] Remde H, Cooper MS, Quinkler M. Successful Asfotase Alfa Treatment in an Adult Dialysis Patient With Childhood-Onset Hypophosphatasia. J Endocr Soc 2017; 1(9): 1188-1193

[20] Genest F, Rak D, Petryk A et al. Physical Function and Health-Related Quality of Life in Adults Treated With Asfotase Alfa for Pediatric-Onset Hypophosphatasia. JBMR Plus 2020: e10395 doi: 10.1002/jbm4.10395

[21] Gospe SM, Santiago-Turla C, DeArmey SM et al. Ectopic Ocular Surface Calcification in Patients With Hypophosphatasia Treated With Asfotase Alfa. Cornea 2019; 38(7): 896-900

[22] Kishnani PS, Rush ET, Arundel P et al. Monitoring guidance for patients with hypophosphatasia treated with asfotase alfa. Mol Genet Metab 2017; 122(1-2): 4-17

[23] Fachinformation Strensiq ${ }^{\circledR}$, S.D.

[24] Schmidt T, Mussawy H, Rolvien T et al. Clinical, radiographic and biochemical characteristics of adult hypophosphatasia. Osteoporos Int 2017; 28(9): 2653-2662

[25] Sebastián-Serrano Á, de Diego-García L, Henshall DC et al. Haploinsufficient TNAP Mice Display Decreased Extracellular ATP Levels and Expression of Pannexin-1 Channels. Front Pharmacol 2018; 9: 170

[26] Sebastián-Serrano Á, Engel T, de Diego-García L et al. Neurodevelopmental alterations and seizures developed by mouse model of infantile hypophosphatasia are associated with purinergic signalling deregulation. Hum Mol Genet 2016; 25(19): 4143-4156

[27] Rush ET, Moseley S, Petryk A. Burden of disease in pediatric patients with hypophosphatasia: Results from the HPP Impact Patient Survey and the HPP Outcomes Study Telephone interview. Orphanet J Rare Dis 2019; 14(1): 201
[28] Weber TJ , Sawyer EK, Moseley S et al. Burden of disease in adult patients with hypophosphatasia: Results from two patient-reported surveys. Metabolism 2016; 65(10): 1522-1530

[29] Michel BA. [Sports in patients with systemic inflammatory musculoskeletal diseases]. Orthopade 1997; 26(11): 972-975

[30] Booth J , Moseley GL, Schiltenwolf M et al. Exercise for chronic musculoskeletal pain: A biopsychosocial approach. Musculoskeletal Care 2017; 15(4): 413-421

[31] Ambrose KR, Golightly YM. Physical exercise as non-pharmacological treatment of chronic pain: Why and when. Best Pract Res Clin Rheumatol 2015; 29(1): 120-130

[32] Kuehn K, Hahn A, Seefried L. Mineral intake and clinical symptoms in adult patients with Hypophosphatasia. J Clin Endocrinol Metab 2020; 105: dgaa324. doi: 10.1210/clinem/dgaa324

[33] Genest F, Seefried L. Klinischer Stellenwert des Serum-Magnesiumwertes und der Supplementation bei Hypophosphatasie. Osteologie 2018; 27: A48-A49

[34] Girschick HJ, Mornet E, Beer M et al. Chronic multifocal non-bacterial osteomyelitis in hypophosphatasia mimicking malignancy. BMC Pediatr 2007; 7: 3

[35] Girschick HJ, Schneider P, Haubitz I et al. Effective NSAID treatment indicates that hyperprostaglandinism is affecting the clinical severity of childhood hypophosphatasia. Orphanet J Rare Dis 2006; 1: 24

[36] Sutton RA, Mumm S, Coburn SP et al. "Atypical femoral fractures" during bisphosphonate exposure in adult hypophosphatasia. J Bone Miner Res 2012; 27(5): 987-994

[37] Whyte MP. Atypical femoral fractures, bisphosphonates, and adult hypophosphatasia. J Bone Miner Res 2009; 24(6): 1132-1134

[38] Genest F, Seefried L. Subtrochanteric and diaphyseal femoral fractures in hypophosphatasia-not atypical at all. Osteoporos Int 2018; 29(8): 1815-1825

[39] Camacho PM, Mazhari AM, Wilczynski C et al. Adult Hypophosphatasia Treated with Teriparatide: Report of 2 Patients and Review of the Literature. Endocr Pract 2016; 22(8): 941-950

[40] Schmidt T, Rolvien T, Linke $C$ et al. Outcome of Teriparatide Treatment on Fracture Healing Complications and Symptomatic Bone Marrow Edema in Four Adult Patients With Hypophosphatasia. JBMR Plus 2019; 3(8): e10215

[41] Whyte MP, Mumm S, Deal C. Adult hypophosphatasia treated with teriparatide. J Clin Endocrinol Metab 2007; 92(4): 1203-1208

[42] Laroche M. Failure of teriparatide in treatment of bone complications of adult hypophosphatasia. Calcif Tissue Int 2012; 90(3): 250

[43] Seefried L, Baumann J, Hemsley $S$ et al. Efficacy of anti-sclerostin monoclonal antibody BPS804 in adult patients with hypophosphatasia. J Clin Invest 2017; 127(6): 2148-2158

[44] Valenza G, Burgemeister S, Girschick $\mathrm{H}$ et al. Analysis of the periodontal microbiota in childhood-type hypophosphatasia. Int J Med Microbiol 2006; 296(7): 493-500

[45] Hofmann C, Girschick HJ, Mentrup B et al. Clinical aspects of hypophsophatasia: an update. Clinic Rev Bone Miner Metab 2013; 11: 60-70

[46] Collmann H, Mornet E, Gattenlöhner $S$ et al. Neurosurgical aspects of childhood hypophosphatasia. Childs Nerv Syst 2009; 25(2): 217-223

[47] Di Rocco F, Baujat G, Cormier-Daire V et al. Craniosynostosis and hypophosphatasia. Arch Pediatr 2017; 24(5s2): 5s89-5s92 